\title{
INSTABILITY, NONEXISTENCE, AND UNIQUENESS IN ELASTICITY WITH POROUS DISSIPATION
}

\author{
M. C. LESEDUARTE AND R. QUINTANILLA \\ Received 18 July 2005; Revised 10 October 2005; Accepted 11 October 2005
}

This paper is devoted to the study of the elasticity with porous dissipation. In the context of the nonlinear problem, we prove instability and nonexistence of solutions. In the context of the linear problem, we obtain exponential growth. We also obtain uniqueness of solutions of the backward in time problem of the linear equations.

Copyright (c) 2006 M. C. Leseduarte and R. Quintanilla. This is an open access article distributed under the Creative Commons Attribution License, which permits unrestricted use, distribution, and reproduction in any medium, provided the original work is properly cited.

\section{Introduction}

In what follows, we consider a theory for the behaviour of porous solids such that the matrix material is elastic and the interstices are void of material; it is a generalization of the classical theory of elasticity. The theory of porous elastic material has been established by Cowin and Nunziato $[2,11]$. In this theory, the bulk density is the product of two scalar fields, the matrix material density and the volume fraction field; it is studied in the book of Ciarletta and Ieşan [1]. Thermal effects were included in the book of Ieşan [4]. Results on linear and nonlinear problems have been obtained recently $[5,14,15]$.

The aim of this paper is the study of the qualitative behaviour of the solutions of the elasticity with porous dissipation. It is worth noting that there are very few contributions to this topic. We can recall some contributions to the nonlinear problem in the book of Ciarletta and Ieșan [1], but they are in the case where porous dissipation is not present. Here we prove instability and nonexistence in the nonlinear case when some conditions on the internal energy and the dissipation function are satisfied. We also work in the linear case and we prove exponential growth of solutions whenever the initial data satisfy several conditions. A uniqueness result is also obtained in the case of the backward in time problem for the linear equations. The results of this paper are of interest from the mechanical and from the mathematical point of view: from the mechanical point of view because we obtain some qualitative results in the theory of elastic materials with voids; from the mathematical point of view because we extend some results that were known 
in the case of no dissipation to the case that there exists dissipation. Here, we are able to overcome the difficulty proposed by this new term.

It is worth recalling that some of the results are inspired by methods and techniques used in the classical elasticity. For example, Section 3 is inspired by the studies of Knops [6]. The methodology of Section 5 has been used by Knops et al. [7], Levine [9], Payne [12], and Knops and Straughan [8]. Recently this approach has been also used by Galaktionov and Pohozaev [3] and Quintanilla [13] has applied it to the thermoelasticity without energy dissipation.

The plan of the paper is as follows. In Section 2, we set down the basic equations and the initial and boundary conditions. An instability result is proved in Section 3. Some cases where the instability assumptions hold are presented in Section 4. In Section 5, we set down a nonexistence result and some cases where the assumptions hold are presented in Section 6. An exponential growth of the solutions for the linear case is proved in Section 7. The paper concludes with a uniqueness result for the backward-in-time problem corresponding to the linear equations.

\section{Preliminaries}

Consider a homogeneous body which occupies a bounded region $B$ that we consider as a reference configuration. The surface $\partial B$ is assumed to have sufficient smoothness to permit applications of the divergence theorem. The particles of the body are identified with their positions in $B$ and we assume that the body is in equilibrium under the action of supply terms and surface tractions. The mechanical motion is described by the displacement $\mathbf{u}$ and the volume fraction $\nu$. We assume that they are referred to as the reference configuration and a fixed system of Cartesian axes. $B$ is at rest relative to the considered system of axes. Equilibrium is destroyed at $t=0$ by instantaneously imparting to all points of $B$ a given displacement and volume fraction, but keeping the boundary fixed throughout the ensuing mechanical motion. The governing equations of the nonlinear elasticity with voids are the equation of motion

$$
\rho \ddot{u}_{i}=T_{K i, K}
$$

the equation

$$
\rho k \ddot{\nu}=H_{K, K}+g \text {, }
$$

and the constitutive equations

$$
T_{K i}=\frac{\partial W}{\partial u_{i, K}}, \quad H_{K}=\frac{\partial W}{\partial \nu_{, K}}, \quad g=-\frac{\partial W}{\partial \nu}+D .
$$

Here $W$ is the internal energy which depends on the gradient of the displacement $u_{i, K}$, the gradient of the volume fraction $\nu_{, K}$, and the volume fraction $\nu$, and $D$ is the dissipation function which also depends on the gradient of the displacement, the gradient of the volume fraction, the volume fraction, and the time derivative of the volume fraction $\dot{\nu}$. 
We assume that $D$ vanishes when $\dot{v}$ vanishes and in general we have the condition

$$
\dot{\nu} \leq 0 \text {. }
$$

If we introduce the constitutive equations into the evolution equations, we obtain the system of equations

$$
\begin{gathered}
\rho \ddot{u}_{i}=\left(\frac{\partial W}{\partial u_{i, K}}\right)_{, K}, \\
\rho k \ddot{\nu}=\left(\frac{\partial W}{\partial \nu_{, K}}\right)_{, K}-\frac{\partial W}{\partial \nu}+D .
\end{gathered}
$$

To the system of field equations we adjoin the initial conditions

$$
\mathbf{u}(\mathbf{x}, 0)=\mathbf{u}^{0}(\mathbf{x}), \quad \dot{\mathbf{u}}(\mathbf{x}, 0)=\mathbf{v}^{0}(\mathbf{x}), \quad \nu(\mathbf{x}, 0)=\nu^{0}(\mathbf{x}), \quad \dot{v}(\mathbf{x}, 0)=\eta^{0}(\mathbf{x}),
$$

and the boundary conditions

$$
\mathbf{u}=0, \quad v=0, \quad \text { on } \partial B
$$

If we multiply (2.5) by $\dot{u}_{i}$ and (2.6) by $\dot{v}$, integrate over $B$, and use the boundary conditions, we obtain the energy equation

$$
E(t)=\frac{1}{2} \int_{B}\left(\rho \dot{u}_{i} \dot{u}_{i}+\rho k(\dot{v})^{2}+2 W\right) d v-\int_{0}^{t} \int_{B} \dot{\nu} D d v d s=E(0) .
$$

Although we work in the case of bounded domains, it is worth noting that the analysis and results of Sections 3, 5, 7, and 8 can be also used in the case of unbounded domains whenever we assume that the solutions vanish at infinity.

\section{Instability: nonlinear case}

The aim of this section is to state and prove the following result.

Theorem 3.1. Let $\left(u_{i}, v\right)$ be a solution of the initial-boundary-value problem (2.5)-(2.8). Let us assume that

$$
\int_{B}\left(\frac{\partial W}{\partial u_{i, K}} u_{i, K}+\frac{\partial W}{\partial \nu_{, K}} v_{, K}+\frac{\partial W}{\partial \nu} v\right) d v \leq 0
$$

and that

$$
\left|\int_{B} v D d v\right| \leq \epsilon \int_{B} \rho k(\dot{\nu})^{2} d v-\int_{B}\left[\frac{\partial W}{\partial u_{i, K}} u_{i, K}+\frac{\partial W}{\partial \nu_{, K}} \nu_{, K}+\frac{\partial W}{\partial \nu} \nu\right] d v,
$$

where $0<\epsilon<1$. Then, the equilibrium state is unstable.

Proof. Define

$$
F(t)=\frac{1}{2} \int_{B}\left(\rho u_{i} u_{i}+\rho k v^{2}\right) d v
$$


4 Elasticity with porous dissipation

Then

$$
\begin{gathered}
\dot{F}(t)=\int_{B}\left(\rho u_{i} \dot{u}_{i}+\rho k v \dot{\nu}\right) d v \\
\ddot{F}(t)=\int_{B}\left(\rho \dot{u}_{i} \dot{u}_{i}+\rho k(\dot{\nu})^{2}\right) d v-\int_{B}\left[\frac{\partial W}{\partial u_{i, K}} u_{i, K}+\frac{\partial W}{\partial \nu_{, K}} v_{, K}+\frac{\partial W}{\partial \nu} v-v D\right] d v .
\end{gathered}
$$

In view of the assumptions, we have

$$
\ddot{F}(t) \geq(1-\epsilon) \int_{B}\left(\rho \dot{u}_{i} \dot{u}_{i}+\rho k(\dot{v})^{2}\right) d v .
$$

Then

$$
F(t) \ddot{F}(t) \geq \frac{(1-\epsilon)}{2} \int_{B}\left(\rho u_{i} u_{i}+\rho k v^{2}\right) d v \int_{B}\left(\rho \dot{u}_{i} \dot{u}_{i}+\rho k(\dot{v})^{2}\right) d v \geq \frac{(1-\epsilon)}{2}[\dot{F}(t)]^{2},
$$

where the last inequality follows from the Schwarz inequality. We see that

$$
\frac{d^{2}}{d t^{2}} F^{\delta}(t) \geq 0
$$

where $\delta=(\epsilon+1) / 2$. So

$$
F^{\delta}(t) \geq \frac{\delta^{-1} F(0)+t(d F / d t(0))}{\delta^{-1} F^{1-\delta}(0)}
$$

which proves that $F^{\delta}(t)$ becomes unbounded whenever $d F / d t(0)>0$.

\section{Instability: discussion}

The energy criterion is sometimes a condition to determine stability or instability. With the help of Theorem 3.1, it is possible to obtain a result when the equilibrium solution corresponds to a maximum of the energy. If we look for one-dimensional examples, we may consider

$$
W=-\left(\left|u_{x}\right|^{p}+\left|v_{x}\right|^{q}+|\nu|^{r}\right), \quad p, q, r>1
$$

Condition (3.1) is satisfied. To satisfy condition (3.2), let us consider functions of the type

$$
D=-d|\nu|^{s} \dot{v}, \quad d>0 .
$$

We have

$$
\begin{aligned}
\left|\int_{B} \nu D d x\right| & \leq d \int_{B}|\nu|^{s+1}|\dot{\nu}| d x \leq d\left(\int_{B}|\nu|^{2(s+1)} d x\right)^{1 / 2}\left(\int_{B}|\dot{\nu}|^{2} d x\right)^{1 / 2} \\
& \leq d\left(\int_{B}\left(\lambda|\nu|^{2(s+1)}+(1-\lambda)\left|\nu_{x}\right|^{2(s+1)} \mu_{2(s+1)}^{2(s+1)}\right) d x\right)^{1 / 2}\left(\int_{B}|\dot{\nu}|^{2} d x\right)^{1 / 2},
\end{aligned}
$$


where

$$
\mu_{2(s+1)}^{-1}=\min _{\phi \in C_{0}^{\infty}(B)} \frac{\left(\int_{B}\left|\phi_{x}\right|^{2(s+1)} d x\right)^{1 /(2(s+1))}}{\left(\int_{B}|\phi|^{2(s+1)} d x\right)^{1 /(2(s+1))}} .
$$

Arithmetic-geometric mean inequality and $\sqrt{a+b} \leq \sqrt{a}+\sqrt{b}$ imply that

$$
\begin{gathered}
\left|\int_{B} \nu D d x\right| \leq \frac{d}{2}\left[\lambda^{1 / 2} \epsilon_{1} \int_{B}|\nu|^{2(s+1)} d x+\mu_{2(s+1)}^{s+1}(1-\lambda)^{1 / 2} \epsilon_{2} \int_{B}\left|\nu_{x}\right|^{2(s+1)} d x\right. \\
\left.+\left(\frac{\lambda^{1 / 2}}{\epsilon_{1}}+\frac{(1-\lambda)^{1 / 2} \mu_{2(s+1)}^{s+1}}{\epsilon_{2}}\right) \int_{B}|\dot{\nu}|^{2} d x\right] .
\end{gathered}
$$

In case that $r=q=2(s+1)$, condition (3.2) is satisfied whenever there are three positive constants $\lambda<1, \epsilon_{1}$, and $\epsilon_{2}$ such that

$$
\begin{gathered}
\lambda^{1 / 2} d \epsilon_{1} \leq 4(s+1), \\
(1-\lambda)^{1 / 2} d \epsilon_{2} \mu_{2(s+1)}^{s+1} \leq 4(s+1), \\
\lambda^{1 / 2} d \epsilon_{2}+(1-\lambda)^{1 / 2} d \epsilon_{1} \mu_{2(s+1)}^{s+1}<2 \rho k \epsilon_{1} \epsilon_{2} .
\end{gathered}
$$

In particular, if $s=0$, the above conditions become

$$
\lambda^{1 / 2} d \epsilon_{1} \leq 4, \quad(1-\lambda)^{1 / 2} d \epsilon_{2} \mu_{2} \leq 4, \quad \lambda^{1 / 2} d \epsilon_{2}+(1-\lambda)^{1 / 2} d \epsilon_{1} \mu_{2}<2 \rho k \epsilon_{1} \epsilon_{2} .
$$

In case that $r=2(s+1)$, but $q \neq 2(s+1)$, condition (3.2) is satisfied whenever there exists a positive constant $\epsilon_{1}$ such that

$$
d \epsilon_{1} \leq 4(s+1), \quad d<2 \rho k \epsilon_{1} .
$$

This last condition can be satisfied whenever $d^{2}<8 \rho k(s+1)$. If $s=0$, the above condition becomes $d^{2}<8 \rho k$.

When $q=2(s+1)$, but $r \neq 2(s+1)$, condition (3.2) is satisfied whenever there exists a positive constant $\epsilon_{2}$ such that

$$
\mu_{2(s+1)}^{s+1} d \epsilon_{2} \leq 4(s+1), \quad \mu_{2(s+1)}^{s+1} d<2 \rho k \epsilon_{2} .
$$

It is satisfied whenever $d^{2} \mu_{2(s+1)}^{2(s+1)}<8 \rho k(s+1)$. If $s=0$, the condition is $d^{2} \mu_{2}^{2}<8 \rho k$.

Another family of examples corresponds to the case when

$$
D=-d\left|u_{x}\right|^{s} \dot{v}
$$

In case that $s / p+1 / r=1 / 2$, we have

$$
\left|\int_{B} \nu D d x\right| \leq d\left(\epsilon_{1}^{p / s} \int_{B}\left|u_{x}\right|^{p} d x\right)^{s / p}\left(\epsilon_{2}^{r} \int_{B}|\nu|^{r} d x\right)^{1 / r}\left(\epsilon_{3}^{2} \int_{B}(\dot{\nu})^{2} d x\right)^{1 / 2},
$$


where $\epsilon_{1} \epsilon_{2} \epsilon_{3}=1$. Thus

$$
\left|\int_{B} \nu D d x\right| \leq \frac{d s \epsilon_{1}^{p / s}}{p} \int_{B}\left|u_{x}\right|^{p} d x+\frac{d \epsilon_{2}^{r}}{r} \int_{B}|\nu|^{r} d x+\frac{d \epsilon_{3}^{2}}{2} \int_{B}(\dot{\nu})^{2} d x .
$$

A sufficient condition to guarantee that condition (3.2) is satisfied could be the existence of three positive constants $\epsilon_{1}, \epsilon_{2}$, and $\epsilon_{3}$ such that $\epsilon_{1} \epsilon_{2} \epsilon_{3}=1$ and

$$
\epsilon_{1}^{p / s} \leq \frac{p^{2}}{d s}, \quad \epsilon_{2}^{r} \leq \frac{r^{2}}{d}, \quad \epsilon_{3}^{2}<\frac{2 \rho k}{d}
$$

In the particular case that $s=1, p=4$, and $r=4$, the previous conditions say

$$
\epsilon_{1}^{4} \leq \frac{16}{d}, \quad \epsilon_{2}^{4} \leq \frac{16}{d}, \quad \epsilon_{3}^{2}<\frac{2 \rho k}{d}
$$

In case that $d^{4}<4^{5} \rho^{2} k^{2}$, we can select $\epsilon_{i}$ satisfying the previous conditions. We consider now $s=0$, so $r=2$. If there are two positive constants $\epsilon_{2}$ and $\epsilon_{3}$ such that $\epsilon_{2} \epsilon_{3}=1$ and $\epsilon_{2}^{2} \leq 4 / d, \epsilon_{3}^{2}<2 \rho k / d$, then condition (3.2) holds. Thus, we obtain the condition $d^{2}<8 \rho k$. We note that it is the same condition as that corresponding to family (4.2) when $s=0$, $r=2$, and $q \neq 2$.

It could also be possible to consider equilibrium states that correspond to a "saddle point" of the inner energy $W$. For instance, we can consider

$$
W=-\left|u_{x}\right|^{p}-\left|v_{x}\right|^{q}+|\nu|^{q} .
$$

Condition (3.1) is satisfied whenever $p, q>1$ and $\mu_{q}<1$. The family defined by (4.2) satisfies condition (3.2) whenever $q=2(s+1)$ and $d^{2} \mu_{2(s+1)}^{2(s+1)}<8 \rho k(s+1)\left(1-\mu_{2(s+1)}^{2(s+1)}\right)$. The case $s=0$ corresponds to $q=2$ and $d^{2} \mu_{2}^{2}<8 \rho k\left(1-\mu_{2}^{2}\right)$.

\section{Nonexistence}

Here, we analyze the consequence of strengthening conditions (3.1) and (3.2). Thus, we assume that condition (3.1) is satisfied and the inequality

$$
\int_{B}\left(\xi W-\frac{\partial W}{\partial u_{i, K}} u_{i, K}-\frac{\partial W}{\partial \nu_{, K}} v_{, K}-\frac{\partial W}{\partial \nu} \nu\right) d v \geq 0
$$

is satisfied for $\xi>2$. Concerning the dissipation function, we assume that

$$
\left|\int_{B} v D d v\right| \leq \epsilon_{1} \int_{B} \rho k(\dot{v})^{2} d v+\int_{B}\left[\xi W-\frac{\partial W}{\partial u_{i, K}} u_{i, K}-\frac{\partial W}{\partial v_{, K}} v_{, K}-\frac{\partial W}{\partial \nu} v\right] d v,
$$

where $(1 / 2)(2+\xi)-\epsilon_{1}=\xi^{*}>2$. 
We define

$$
G_{\beta, t_{0}}=F(t)+\frac{1}{2} \beta\left(t+t_{0}\right)^{2}, \quad \beta \geq 0,
$$

where $F(t)$ is defined in (3.3). We have

$$
\begin{gathered}
\dot{G}_{\beta, t_{0}}=\dot{F}(t)+\beta\left(t+t_{0}\right), \\
\ddot{G}_{\beta, t_{0}}=\ddot{F}(t)+\beta .
\end{gathered}
$$

In view of the energy equation, we have that

$$
\begin{aligned}
\ddot{G}_{\beta, t_{0}} \geq & \frac{1}{2}(2+\xi) \int_{B}\left(\rho \dot{u}_{i} \dot{u}_{i}+\rho k(\dot{\nu})^{2}\right) d v \\
& +\int_{B}\left[\xi W-\frac{\partial W}{\partial u_{i, K}} u_{i, K}-\frac{\partial W}{\partial \nu_{, K}} \nu_{, K}-\frac{\partial W}{\partial \nu} \nu+\nu D\right] d v-(\xi E(0)-\beta)
\end{aligned}
$$

Inequality (5.2) implies that

$$
\ddot{G}_{\beta, t_{0}} \geq \xi^{*} \int_{B}\left(\rho \dot{u}_{i} \dot{u}_{i}+\rho k(\dot{v})^{2}\right) d v-(\xi E(0)-\beta) .
$$

Schwarz's inequality leads to

$$
G_{\beta, t_{0}} \ddot{G}_{\beta, t_{0}}-\frac{\xi^{*}}{2}\left(\dot{G}_{\beta, t_{0}}\right)^{2} \geq-\left(\xi E(0)+\left(\xi^{*}-1\right) \beta\right) G_{\beta, t_{0}} .
$$

Since $\xi^{*}>2$, the last inequality may be put in the form

$$
\frac{d^{2} G_{\beta, t_{0}}^{-\gamma}}{d t^{2}} \leq \gamma G_{\beta, t_{0}}^{-(1+\gamma)}\left(\xi E(0)+\left(\xi^{*}-1\right) \beta\right),
$$

where

$$
\gamma=\frac{\xi^{*}-2}{2}
$$

If we choose $\beta$ such that $\xi E(0)+\left(\xi^{*}-1\right) \beta=0$, inequality (5.8) implies that

$$
\frac{d^{2} G_{\beta, t_{0}}^{-\gamma}}{d t^{2}} \leq 0
$$

This shows that $G_{\beta, t_{0}}^{-\gamma}$ is a concave function. We have

$$
G_{\beta, t_{0}}(t) \geq G_{\beta, t_{0}}(0)\left[1-\gamma t \frac{d G_{\beta, t_{0}}}{d t}(0) G_{\beta, t_{0}}(0)^{-1}\right]^{-1 / \gamma} .
$$

We can always select $t_{0}$ sufficiently large to guarantee that the derivative of $G_{\beta, t_{0}}$ is positive at zero. This provides a lower bound to $G_{\beta, t_{0}}$ which becomes unbounded in finite time. 
An integration of the inequality (5.10) also gives the estimate

$$
G_{\beta, t_{0}}^{\gamma}(t) \leq \frac{G_{\beta, t_{0}}^{\gamma}(0) G_{\beta, t_{0}}^{\gamma}(T)}{(1-t / T) G_{\beta, t_{0}}^{\gamma}(T)+(t / T) G_{\beta, t_{0}}^{\gamma}(0)},
$$

where we assume that the solution exists in $0 \leq t \leq T$. From (5.12), we may deduce the uniqueness of the null solution, and also continuous dependence upon the initial data.

\section{Nonexistence: discussion}

In this section, we give some examples where conditions (5.1) and (5.2) are satisfied. In fact, we discuss when the examples proposed in Section 4 also satisfy conditions (5.1) and (5.2). First, we see that the family (4.1) with functions of type (4.2) satisfy condition (5.1) whenever $p, q$, and $r$ are greater than two. Thus, in this section, we consider the function $W$ defined in (4.1) when the parameters $p, q$, and $r$ satisfy the previous condition. In this case,

$$
\xi W-\left(\frac{\partial W}{\partial u_{x}} u_{x}+\frac{\partial W}{\partial \nu_{x}} v_{x}+\frac{\partial W}{\partial \nu} \nu\right)=(p-\xi)\left|u_{x}\right|^{p}+(q-\xi)\left|\nu_{x}\right|^{q}+(r-\xi)|\nu|^{r} .
$$

We can take $\xi$ as near as we want to the minimum of $p, q, r$. If we assume that $r=q=$ $2(s+1)$, the assumptions are satisfied whenever there exist positive numbers $\lambda<1, \xi^{*}>2$, $\epsilon_{2}$, and $\epsilon_{3}$ such that

$$
\begin{gathered}
\lambda^{1 / 2} d \epsilon_{2} \leq 4(s+1)-2 \xi, \quad(1-\lambda)^{1 / 2} d \epsilon_{3} \mu_{2(s+1)}^{s+1} \leq 4(s+1)-2 \xi, \\
\lambda^{1 / 2} d \epsilon_{3}+(1-\lambda)^{1 / 2} d \epsilon_{2} \mu_{2(s+1)}^{s+1} \leq 2 \rho k \epsilon_{2} \epsilon_{3}\left[\frac{1}{2}(2+\xi)-\xi^{*}\right] .
\end{gathered}
$$

In case that $r=2(s+1)$, but $q \neq 2(s+1)$, condition (3.2) is satisfied whenever there exist two positive constants $\epsilon_{2}, \xi^{*}>2$ such that

$$
d \epsilon_{2} \leq 4(s+1)-2 \xi, \quad d \leq 2 \rho k\left[\frac{1}{2}(2+\xi)-\xi^{*}\right] \epsilon_{2}
$$

We note that if $s=0$, then we have the condition $d^{2} \leq 4 \rho k(2-\xi)\left[(1 / 2)(2+\xi)-\xi^{*}\right]$.

Perhaps, it would be good to give an explicit example. For instance, let us assume that $r=3, s=1 / 2$, and $q, p$ are greater than three. We can take $\xi=2.5$. The condition is the existence of two positive constants $\epsilon_{1}$ and $\xi^{*}>2$ such that

$$
d \epsilon_{1} \leq 1, \quad d \leq 2 \rho k\left[2.25-\xi^{*}\right] \epsilon_{1} .
$$

If we select $\xi^{*}=2.05$, our second condition becomes

$$
d \leq 0.4 \rho k \epsilon_{1} .
$$

We can select $\epsilon_{1}$ satisfying the required conditions whenever

$$
d^{2} \leq 0.4 \rho k
$$


It is also possible to study conditions corresponding to the family of dissipation functions defined in (4.10). It is possible as well to consider the case when the function $W$ is defined by $(4.15)$.

\section{Exponential growth: linear case}

In this section, we consider the problem determined by a centrosymmetric porous elastic material. To make the calculations easier, we also assume that the material is linearly homogeneous. In this case, the system of equations can be expressed as

$$
\begin{gathered}
\rho \ddot{u}_{i}=C_{i j r s} u_{i, j r}+B_{i j} \nu_{, j}, \\
\rho k \ddot{\nu}=A_{i j} \nu_{, i j}-B_{i j} u_{i, j}-\eta \nu-\tau \dot{\nu} .
\end{gathered}
$$

First we assume that $\rho, k$, and $\tau$ are strictly positive, but we do not impose the positivity of the internal energy. However, at the end of this section, we will consider the case $k=0$. In fact, the aim of this section is to prove that whenever the internal energy is negative, then there exist solutions that grow exponentially.

We will work with the functional

$$
H_{\beta, t_{0}}(t)=G_{\beta, t_{0}}(t)+\frac{\tau}{2} \int_{B} \int_{0}^{t} v^{2} d s d v
$$

We observe by differentiation that

$$
\begin{gathered}
\dot{H}_{\beta, t_{0}}(t)=\dot{G}_{\beta, t_{0}}(t)+\tau \int_{B} \int_{0}^{t} v \dot{v} d s d v+\frac{\tau}{2} \int_{B}\left(\nu^{0}\right)^{2} d v, \\
\ddot{H}_{\beta, t_{0}}(t)=\ddot{G}_{\beta, t_{0}}(t)+\tau \int_{B} v \dot{v} d v .
\end{gathered}
$$

In the linear case, the energy equation (2.9) becomes

$$
\begin{gathered}
E(t)=\frac{1}{2} \int_{B}\left(\rho \dot{u}_{i} \dot{u}_{i}+\rho k(\dot{\nu})^{2}+C_{i j r s} u_{i, j} u_{s, r}+2 B_{i j} u_{i, j} \nu+A_{i j} \nu_{, i} \nu_{, j}+\eta v^{2}\right. \\
\left.+2 \tau \int_{0}^{t}(\dot{v})^{2} d s\right) d v=E(0)
\end{gathered}
$$

Also, multiplication of (7.1) by $u_{i}$ and (7.2) by $\nu$ and integration over $B$ yields

$$
\begin{gathered}
\int_{B}\left(\rho u_{i} \ddot{u}_{i}+C_{i j r s} u_{i, j} u_{s, r}+B_{i j} u_{i, j} \nu\right) d v=0, \\
\int_{B}\left(\rho k \nu \ddot{v}+A_{i j} \nu_{, i} \nu_{, j}+B_{i j} u_{i, j} \nu+\eta \nu^{2}\right) d v=-\tau \int_{B} \dot{\nu} \nu d v .
\end{gathered}
$$

Thus, we obtain

$$
\ddot{H}_{\beta, t_{0}}=2 \int_{B}\left(\rho \dot{u}_{i} \dot{u}_{i}+\rho k \dot{\nu} \dot{v}+\tau \int_{0}^{t}(\dot{v})^{2} d s\right) d v-(2 E(0)-\beta) .
$$


Hence,

$$
H_{\beta, t_{0}} \ddot{H}_{\beta, t_{0}}-\left(\dot{H}_{\beta, t_{0}}-\frac{\omega}{2}\right)^{2} \geq 0,
$$

if

$$
\beta=-2 E(0)
$$

and

$$
\omega=2 \tau \int_{B}\left(\nu^{0}\right)^{2} d \nu
$$

If we take $t_{0}$ such that

$$
\dot{H}_{\beta, t_{0}}(0)>\omega \text {, }
$$

it may be proved that

$$
H_{\beta, t_{0}} \geq \frac{H_{\beta, t_{0}}(0) \dot{H}_{\beta, t_{0}}(0)}{\dot{H}_{\beta, t_{0}}(0)-\omega} \exp \left(\frac{\dot{H}_{\beta, t_{0}}(0)-\omega}{H_{\beta, t_{0}}(0)}\right) t-\frac{\omega H_{\beta, t_{0}}(0)}{\dot{H}_{\beta, t_{0}}(0)-\omega} .
$$

If we consider the function $H_{0,0}$, estimate (7.3) implies that

$$
H_{0,0}(t) \geq \frac{H_{\beta, t_{0}}(0) \dot{H}_{\beta, t_{0}}(0)}{\dot{H}_{\beta, t_{0}}(0)-\omega} \exp \left(\frac{\dot{H}_{\beta, t_{0}}(0)-\omega}{H_{\beta, t_{0}}(0)}\right) t-\frac{\omega H_{\beta, t_{0}}(0)}{\dot{H}_{\beta, t_{0}}(0)-\omega}-\frac{\beta}{2}\left(t+t_{0}\right)^{2} .
$$

We have proved the following.

Theorem 7.1. Let $\left(u_{i}, v\right)$ be a solution of the initial-boundary-value problem determined by (2.1)-(2.6), such that the initial conditions satisfy that $E(0)<0$. Then, for $\beta$ satisfying (7.9) and $t_{0}$ determined from (7.10) and (7.11), it follows that for large time the function $H_{0,0}$ grows exponentially.

Theorem 7.1 says that whenever the initial conditions are such that $E(0)<0$, the solution grows exponentially. In view of the assumptions on constitutive tensors, we can obtain solutions with negative energy.

The quasistatic evolution of microvoids can be considered (see [10]). From a mathematical point of view, it corresponds to the case where we assume that $k=0$ in (7.2). Then, we obtain system of (7.1) and the equation

$$
\tau \dot{v}=A_{i j} \nu_{, i j}-B_{i j} u_{i, j}-\eta \nu .
$$

As far as the authors know, this system has not been studied in the literature. However, it is easy to obtain uniqueness and exponential growth of solutions in a similar way as for the system (7.1)-(7.2). To prove uniqueness, we can define the function

$$
J(t)=\frac{1}{2} \int_{B}\left(\rho u_{i} u_{i}+\tau \int_{0}^{t} v^{2} d s\right) d v .
$$


When we assume null initial conditions and boundary conditions (2.8), we see that the function $J(t)$ satisfies the inequality (7.8), where $\omega=0$. This inequality is well known and it implies that (see [6])

$$
J(t) \leq J(0)^{1-t / T} J(T)^{t / T}
$$

for $0 \leq t \leq T$. This estimate implies that $J(t)=0$ whenever we assume null initial condition and then the uniqueness of solutions follows.

Also, it is possible to prove the growth exponential of solutions of the problem determined by (7.2) and (7.14). To this end, we consider the function

$$
J_{\beta, t_{0}}(t)=J(t)+\frac{\beta}{2}\left(t+t_{0}\right)^{2}
$$

It is worth noting that in this case the energy of the system is given by

$$
E_{1}(t)=\frac{1}{2} \int_{B}\left(\rho \dot{u}_{i} \dot{u}_{i}+C_{i j r s} u_{i, j} u_{s, r}+2 B_{i j} u_{i, j} \nu+A_{i j} \nu_{, i} \nu_{, j}+\eta v^{2}+2 \tau \int_{0}^{t}(\dot{\nu})^{2} d s\right) d v=E_{1}(0)
$$

The analysis proposed previously shows that $J_{\beta, t_{0}}(t)$ satisfies (7.8) and then the exponential growth of solutions is obtained whenever $E_{1}(0)<0$.

\section{Uniqueness backward in time problem: linear case}

In this section, we consider the problem determined by the backward in time problem for a linear porous elastic material. We continue with the assumptions proposed in Section 7 with respect to $\rho, k$, and $\tau$, but we do not impose any condition neither on the positivity nor on the negativity of the internal energy. Thus, the internal energy has not a defined sign (which is the most general case). Thus we consider a homogeneous and centrosymmetric porous elastic material. In this case, the system of equations can be expressed as

$$
\begin{gathered}
\rho \ddot{u}_{i}=C_{i j r s} u_{j, r s}+B_{i j} \nu_{, j}, \\
\rho k \ddot{\nu}=A_{i j} \nu_{, i j}-B_{i j} u_{i, j}-\eta \nu+\tau \dot{\nu} .
\end{gathered}
$$

To treat the uniqueness question, let $\left(u_{i}, v\right)$ be the difference of two solutions that satisfy the same initial and boundary conditions. Thus $\left(u_{i}, v\right)$ satisfies the system $(8.1)$, with the boundary conditions (2.8) and the homogeneous initial conditions

$$
u_{i}(\mathbf{x}, 0)=v(\mathbf{x}, 0)=0, \quad \text { on } B .
$$


12 Elasticity with porous dissipation

We can form the identities

$$
\begin{aligned}
& \int_{0}^{t} \int_{B} \rho \ddot{u}_{i}(s) \dot{u}_{i}(2 t-s) d v d s \\
& =-\int_{0}^{t} \int_{B} C_{i j r s} u_{i, j}(s) \dot{u}_{s, r}(2 t-s) d v d s-\int_{0}^{t} \int_{B} B_{i j} v(s) \dot{u}_{i, j}(2 t-s) d v d s, \\
& \int_{0}^{t} \int_{B} \rho \ddot{u}_{i}(2 t-s) \dot{u}_{i}(s) d v d s \\
& =-\int_{0}^{t} \int_{B} C_{i j r s} u_{i, j}(2 t-s) \dot{u}_{s, r}(s) d v d s-\int_{0}^{t} \int_{B} B_{i j} v(2 t-s) \dot{u}_{i, j}(s) d v d s, \\
& \int_{0}^{t} \int_{B} \rho k \ddot{\nu}(s) \dot{\nu}(2 t-s) d v d s \\
& =-\int_{0}^{t} \int_{B} A_{i j} v_{, i}(s) \dot{v}_{, j}(2 t-s) d v d s-\int_{0}^{t} \int_{B} B_{i j} u_{i, j}(s) \dot{v}(2 t-s) d v d s \\
& -\int_{0}^{t} \int_{B} \eta v(s) \dot{v}(2 t-s) d v d s+\int_{0}^{t} \int_{B} \tau \dot{\nu}(s) \dot{\nu}(2 t-s) d v d s \\
& \int_{0}^{t} \int_{B} \rho k \ddot{\nu}(2 t-s) \dot{v}(s) d v d s \\
& =-\int_{0}^{t} \int_{B} A_{i j} \nu_{, i}(2 t-s) \dot{v}_{, j}(s) d v d s-\int_{0}^{t} \int_{B} B_{i j} u_{i, j}(2 t-s) \dot{v}(s) d v d s \\
& -\int_{0}^{t} \int_{B} \eta v(2 t-s) \dot{v}(s) d v d s \int_{0}^{t} \int_{B} \tau \dot{\nu}(2 t-s) \dot{v}(s) d v d s .
\end{aligned}
$$

If we recall that $\dot{f}(2 t-s)=-(d / d s) f(2 t-s)$ and form the combinations (8.3)-(8.4)+ (8.5)-(8.6) after some integrations, one finds

$$
\int_{B}\left(\rho \dot{u}_{i} \dot{u}_{i}+\rho k(\dot{v})^{2}-C_{i j r s} u_{i, j} u_{s, r}-2 B_{i j} u_{i, j} \nu-A_{i j} \nu_{, i} \nu_{, j}-\eta v^{2}\right) d v=0 .
$$

We can obtain the energy equation

$$
\begin{aligned}
E(t)= & \frac{1}{2} \int_{B}\left(\rho \dot{u}_{i} \dot{u}_{i}+\rho k(\dot{\nu})^{2}+C_{i j r s} u_{i, j} u_{s, r}+2 B_{i j} u_{i, j} \nu+A_{i j} v_{, i} \nu_{, j}+\eta v^{2}\right) d v \\
& -\tau \int_{0}^{t} \int_{B}(\dot{\nu})^{2} d v d s=0 .
\end{aligned}
$$

Through combination of the two last equalities, we find that

$$
\int_{B}\left(\rho \dot{u}_{i} \dot{u}_{i}+\rho k(\dot{v})^{2}-\tau \int_{0}^{t}(\dot{v})^{2} d s\right) d v=0 .
$$

If we consider the function

$$
Z(t)=\int_{B}\left(\rho \dot{u}_{i} \dot{u}_{i}+\rho k(\dot{v})^{2}\right) d v=\tau \int_{0}^{t} \int_{B}(\dot{v})^{2} d v d s,
$$

we can compute a positive constant such that

$$
Z^{\prime}(t) \leq C Z(t)
$$


that after a quadrature implies that

$$
Z(t) \leq Z(0) \exp (C t)
$$

As we assume homogeneous initial conditions, it follows that $Z(0)=0$. Then, $Z(t)=0$ for all time and we obtain the uniqueness of solutions.

\section{Acknowledgment}

This work is part of the project "Aspectos de estabilidad en termomecánica"(BFM/FEDER 2003-00309).

\section{References}

[1] M. Ciarletta and D. Ieşan, Non-Classical Elastic Solids, Pitman Research Notes in Mathematics Series, vol. 293, Longman Scientific \& Technical, Harlow; John Wiley \& Sons, New York, 1993.

[2] S. C. Cowin and J. W. Nunziato, Linear elastic materials with voids, Journal of Elasticity 13 (1983), no. $2,125-147$.

[3] V. A. Galaktionov and S. I. Pohozaev, Blow-up and critical exponents for nonlinear hyperbolic equations, Nonlinear Analysis. Theory, Methods \& Applications. Series A: Theory and Methods 53 (2003), no. 3-4, 453-466.

[4] D. Ieşan, Thermoelastic Models of Continua, Solid Mechanics and Its Applications, vol. 118, Kluwer Academic, Dordrecht, 2004.

[5] D. Ieşan and R. Quintanilla, Decay estimates and energy bounds for porous elastic cylinders, Zeitschrift für Angewandte Mathematik und Physik (ZAMP) 46 (1995), no. 2, 268-281.

[6] R. J. Knops, Instability and the ill-posed Cauchy problem in elasticity, Mechanics of Solids (H. G. Hopkins and M. J. Sewell, eds.), Pergamon Press, New York, 1982, pp. 357-382.

[7] R. J. Knops, H. A. Levine, and L. E. Payne, Non-existence, instability, and growth theorems for solutions of a class of abstract nonlinear equations with applications to nonlinear elastodynamics, Archive for Rational Mechanics and Analysis 55 (1974), 52-72.

[8] R. J. Knops and B. Straughan, Non-existence of global solutions to non-linear Cauchy problems arising in mechanics, Trends in Applications of Pure Mathematics to Mechanics (Conf., Univ. Lecce, Lecce, 1975), Monographs and Studies in Math., vol. 2, Pitman, London, 1976, pp. 187206.

[9] H. A. Levine, Instability and nonexistence of global solutions to nonlinear wave equations of the form $P u_{t t}=-A u+\mathscr{F}(u)$, Transactions of the American Mathematical Society 192 (1974), 121.

[10] M. Mosconi, A variational approach to porous elastic bodies, Zeitschrift für Angewandte Mathematik und Physik (ZAMP) 56 (2005), no. 3, 548-558.

[11] J. W. Nunziato and S. C. Cowin, A nonlinear theory of elastic materials with voids, Archive for Rational Mechanics and Analysis 72 (1979/1980), no. 2, 175-201.

[12] L. E. Payne, Improperly Posed Problems in Partial Differential Equations, Regional Conference Series in Applied Mathematics, no. 22, SIAM, Pennsylvania, 1975.

[13] R. Quintanilla, Instability and non-existence in the nonlinear theory of thermoelasticity without energy dissipation, Continuum Mechanics and Thermodynamics 13 (2001), no. 2, 121-129.

[14] _ On uniqueness and continuous dependence in the nonlinear theory of mixtures of elastic solids with voids, Mathematics and Mechanics of Solids 6 (2001), no. 3, 281-298. 


\section{Elasticity with porous dissipation}

[15] Slow decay for one-dimensional porous dissipation elasticity, Applied Mathematics Letters 16 (2003), no. 4, 487-491.

M. C. Leseduarte: Departament de Matemàtica Aplicada 2, Universitat Politècnica de Catalunya, Colom 11, 08222 Terrassa, Barcelona, Spain

E-mail address: mari.carme.leseduarte@upc.edu

R. Quintanilla: Departament de Matemàtica Aplicada 2, Universitat Politècnica de Catalunya, Colom 11, 08222 Terrassa, Barcelona, Spain

E-mail address: ramon.quintanilla@upc.edu 


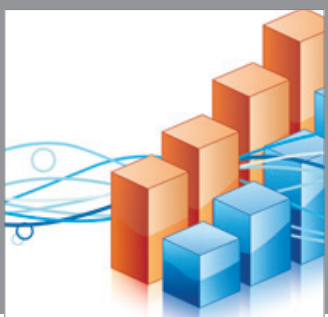

Advances in

Operations Research

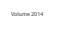

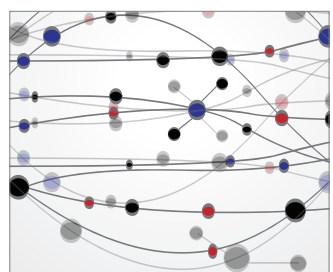

\section{The Scientific} World Journal
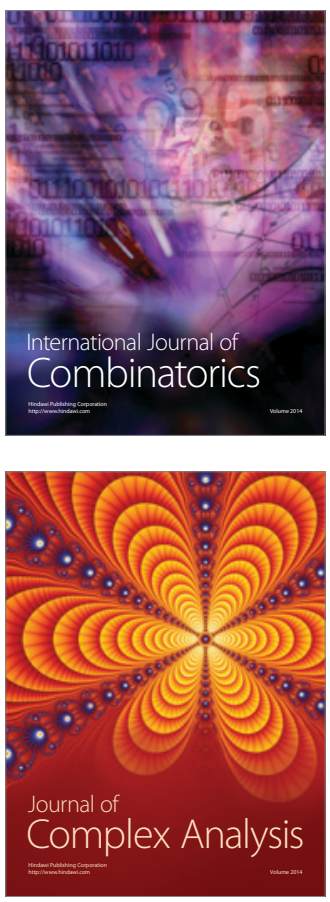

International Journal of

Mathematics and

Mathematical

Sciences
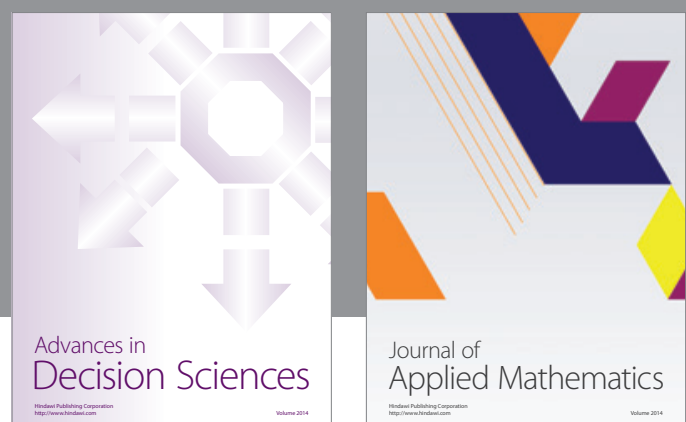

Journal of

Applied Mathematics
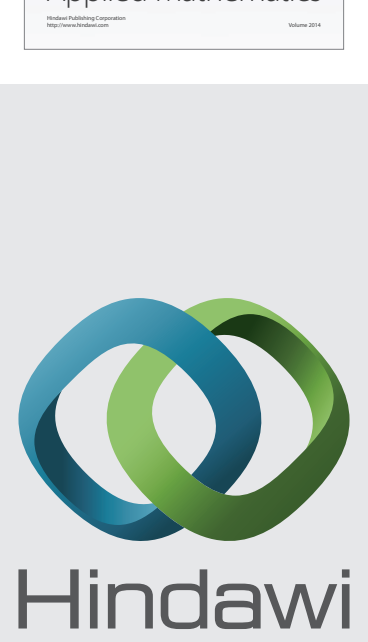

Submit your manuscripts at http://www.hindawi.com
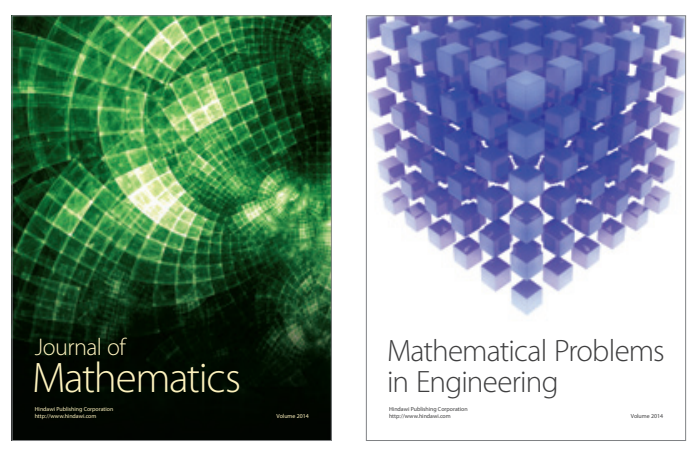

Mathematical Problems in Engineering
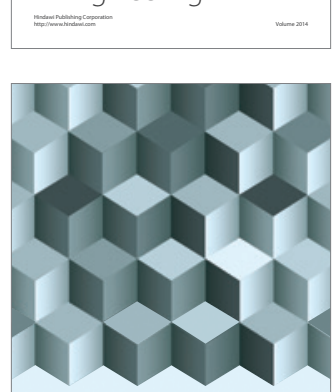

Journal of

Function Spaces
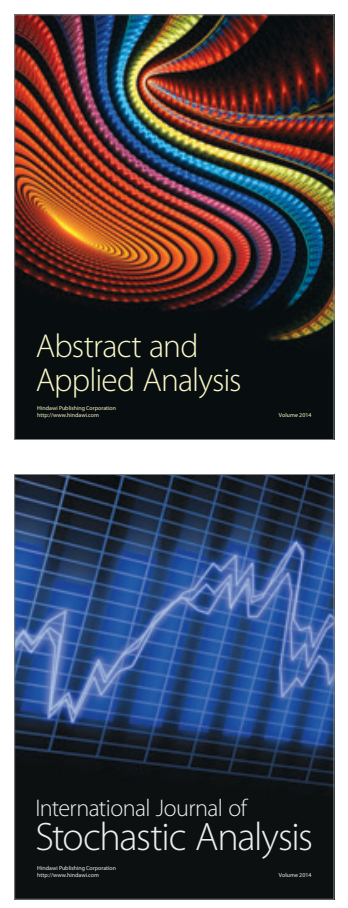

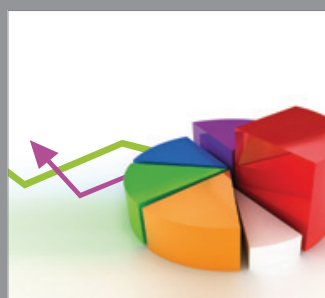

ournal of

Probability and Statistics

Promensencen
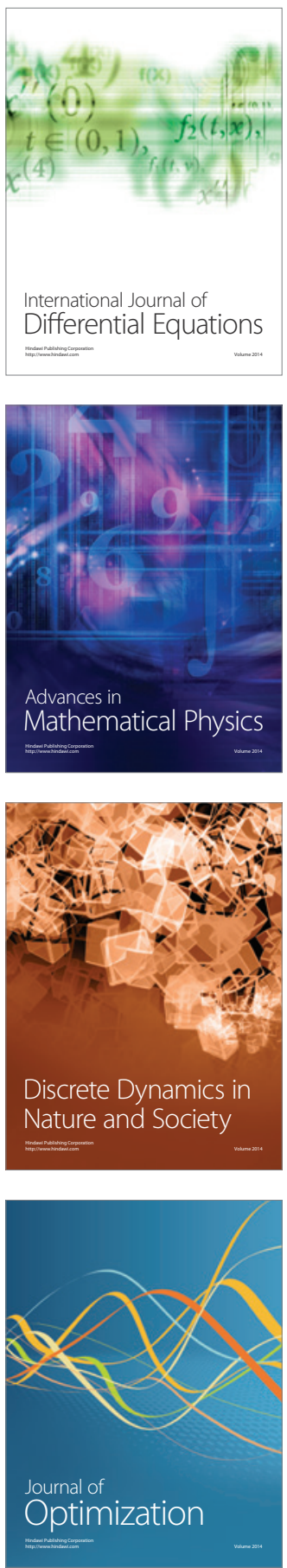